\title{
Intramural Hematoma of the Pulmonary Artery and Hemopneumomediastinum after Endobronchial Ultrasound-Guided Transbronchial Needle Aspiration
}

\author{
Maribel Botana-Rial Manuel Núñez-Delgado Abel Pallarés-Sanmartín \\ Virginia Leiro-Fernández Cristina Represas Represas Ana Isabel González Silva \\ Alberto Fernández-Villar \\ Bronchopleural Techniques Unit, Pulmonology Service, Respiratory and Infectious Disease Research Group, \\ Biomedical Research Institute, Complexo Hospitalario Universitario de Vigo, Vigo, Spain
}

\section{Established Facts}

- Complications related to endobronchial ultrasound-guided transbronchial needle aspiration are rare.

\section{Novel Insights}

- Endobronchial ultrasound-guided transbronchial needle aspiration of small nodes can lead to an intramural hematoma of the pulmonary vessels and pneumomediastinum.

\section{Key Words}

Endobronchial ultrasound · Transbronchial needle aspiration · Hematoma • Hemopneumomediastinum • Pulmonary artery

\begin{abstract}
Endobronchial ultrasound-guided transbronchial needle aspiration (EBUS-TBNA) is a safe and effective technique for the sampling of mediastinal and hilar adenopathies. We describe the first reported case of pulmonary artery intramural hematoma after EBUS-TBNA puncture of this artery. The complication was visualized by ultrasound and resolved spontaneously.

Copyright $\odot 2011$ S. Karger AG, Basel
\end{abstract}

\section{Introduction}

Endobronchial ultrasound-guided transbronchial needle aspiration (EBUS-TBNA) allows the sampling of tracheobronchial wall-adjacent lymph nodes under direct visual control in real time $[1,2]$. Recent systematic reviews summarized the strong diagnostic accuracy and safety of this technique [3]. Complications of EBUSTBNA are similar to those of conventional TBNA. Pneumomediastinum, mediastinitis, bleeding due to the puncturing of large mediastinal vessels [1-3] and rare complications have been reported [4]. So far, complications of the pneumothorax [3], several cases of infection $[5,6]$ and airway alterations [7] have been reported in ex-

\section{KARGER \\ Fax +41613061234 E-Mail karger@karger.ch} www.karger.com
Alberto Fernández-Villar

Pulmonology Service, Complexo Hospitalario Universitario de Vigo

C/Pizarro 22

ES-36204 Vigo, Pontevedra (Spain)

Tel. +34 696826 015, E-Mail alberto.fernandez.villar@sergas.es 

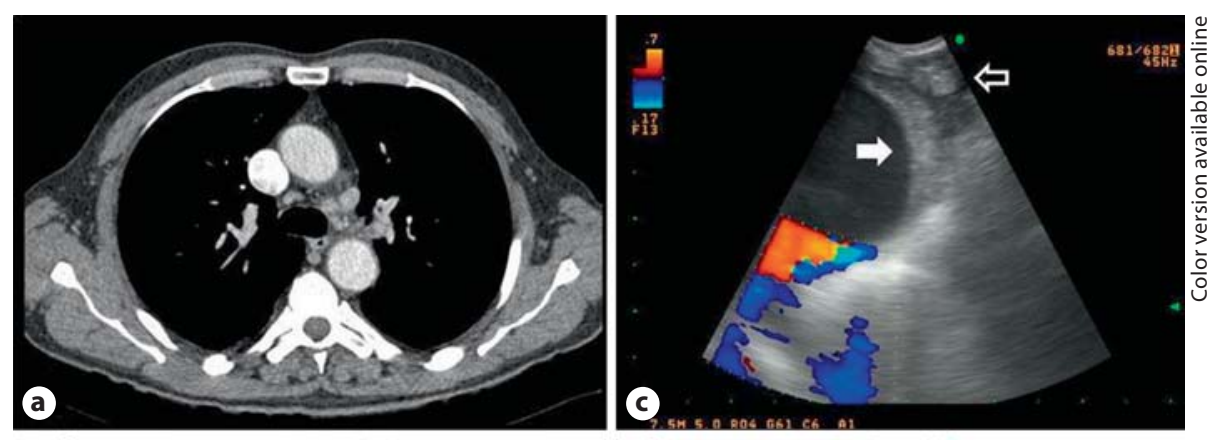

Fig. 1. a Thoracic CT imaging shows small lymph nodes at the left paratracheal station. b Linear echobronchoscope image of the puncture needle within the $4 \mathrm{~L}$ lymph node and its dimensions. c, d Linear echobronchoscope images of a crescent on the upper surface of the PA (compact arrow) and adenopathy (empty arrow).
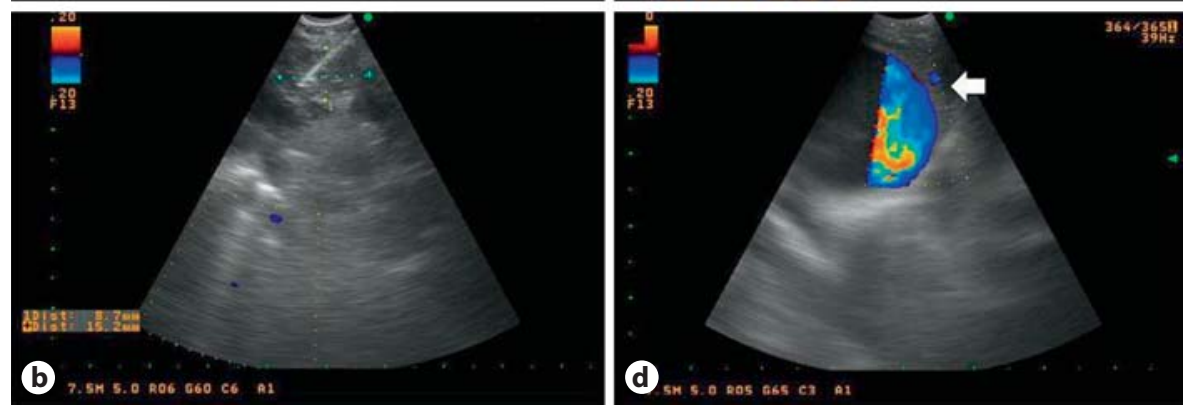

ceptional cases. However, pneumomediastinum and bleeding due to the puncture of a large hilar or mediastinal vessel have not been reported. We describe the first documented case of an intramural hematoma of the pulmonary artery (PA) and a secondary hemopneumomediastinum.

\section{Case Report}

A 62-year-old man presented with a history of left chest pain that increased with movement and a loss of weight and appetite. He had a medical history of hypertension and hyperlipidemia (controlled with diet and drug therapy), and 30 pack-years of smoking. The physical examination and blood analysis were normal. Computed tomography (CT) showed a 28 -millimeter left upper lobe nodule with left paratracheal and aortopulmonary lymph nodes with a diameter less than $10 \mathrm{~mm}$ (fig. 1a). An EBUS-TBNA was performed with the intention of diagnosing and staging the probable lung tumor. An XBF-UC180F echobronchoscope (Olympus Optical Co. Ltd., Tokyo, Japan) was employed. The procedure was monitored with the patient under conscious sedation (midazolam and fentanyl) and local anesthesia with $2 \%$ lidocaine. A sonographic examination was performed first. Some hypoechoic hilar and mediastinal structures, without internal color Doppler flow, compatible with a lymph node of the 11L (with a minor axis diameter of $12 \mathrm{~mm}$ ) and $4 \mathrm{~L}$ node stations (with a minor axis diameter of $9 \mathrm{~mm}$ ) were identified (fig. 1b). The aortopulmonary window adenopathy was not clearly visualized. For this reason, we decided to perform a TBNA of the 4L lymph node with an NA2015X-4022 needle
(Olympus Optical) (fig. 1b). On the first attempt, the needle was accidentally introduced about $2 \mathrm{~mm}$ into the PA. The needle was removed from the PA, placed inside the lymph node, and vacuum aspiration was performed. The pathologist reported that the sample was compatible with non-small cell carcinoma at onsite cytological assessment. Subsequently, a sonographic crescent image at the top of the PA was observed (fig. 1c, d). This image was compatible with a small intramural hematoma of the PA. The injury was observed sonographically for several minutes, and after ensuring no further development of the hematoma, the procedure was suspended. After the procedure, the patient was asymptomatic with no alterations in vital signs. An angio-CT was immediately performed (fig. 2) showing a 5-millimeterwall-thickening at the level of the medial and superior PA face that extended from its origin to its bifurcation. No contrast outside the vessel was identified in the arterial phase, but a small enhancing collection was identified extending caudally, originating in the aortopulmonary window and compatible with mediastinal bleeding. In addition, there was a small pneumomediastinum in the left margin near the trachea and the left main bronchus. The patient was hospitalized for observation. CT performed $24 \mathrm{~h}$ later showed a clear improvement of the lesions described above and the patient was discharged.

\section{Discussion}

EBUS-TBNA is a safe technique. Due to its widespread use, however, rare cases of complications do get reported, specifically those associated with puncture attempts from the airway, including airway wall damage [7], and 
Fig. 2. a, b Thoracic CT axial images of the medial and superior artery wall thickening and a small mediastinal collection. c Coronal section of the same findings and a small paratracheal pneumomediastinum. A nodular lesion in the left upper lobe and paratracheal and aortopulmonary window adenopathies can also be seen.
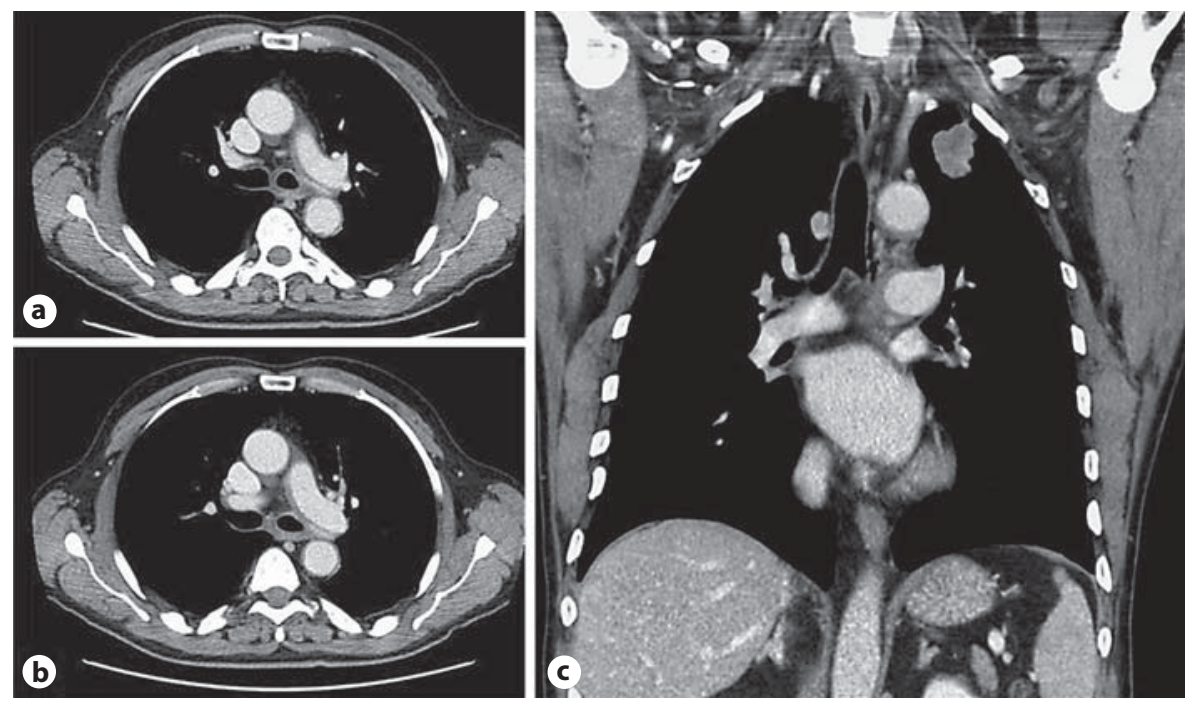

those occurring due to the introduction of microorganisms into the mediastinum $[5,6]$. Although the complications reported here have been seen with conventional TBNA, this is the first published case of their occurrence with EBUS-TBNA. Tissue sampling under direct visual control in real time allowed visualization of the airwayadjacent vessels, decreasing the chance of accidental puncture. Puncture needles included a security system that determined the length of the needle to be withdrawn, which could also be calculated on the ultrasound image by measuring the distance from the lymph node center to the needle's entry point into the tracheal wall [1]. However, in some nodal regions, respiratory movements and arterial pulse forced the explorer to pace himself with these movements, in particular with lymph nodes of small diameter. If the measurement of the length of needle to be withdrawn is not very accurate, as in our case, there is a potential for accidental vessel puncture. If this occurs, the needle should be immediately withdrawn, avoiding aspiration inside the vessel. As shown in figure $1 \mathrm{c}$ and $\mathrm{d}$, an echobronchoscope allowed visualization of these complications, demonstrating the intramural hematoma of the vessel wall.

The puncture of large vessels with other diagnostic techniques is relatively common $[8,9]$. Vincent et al. [8] reported the intentional puncture of a left hilar mass through the PA without producing any complication, arguing for the safety of the technique with low-pressure vessels. Recently, von Bartheld et al. [9] published a series of 14 cases of para-aortic lymph node or mass punctures through this vessel, with 22-gauge needles led by endo- scopic ultrasonography, where only 2 cases of para-aortic hematomas occurred. Both were diagnosed immediately and confirmed by ultrasound and CT. We do not know why the PA puncture in our patient produced the described complications, although one reason could be that the respiratory movements or coughing typical of EBUS (not of endoscopic ultrasonography), combined with the pulsations of the PA and aorta, increased the degree of vessel wall laceration. It is likely that due to the imaging of the PA wall crescent image, we were made aware of the possibility of the other complications. The PA intramural hematoma was detected by EBUS and a small left paratracheal and aortopulmonary hemopneumomediastinum was visualized by CT. If this hematoma had not been seen, we would not have investigated and recognized the subsequent complications. It is also likely that if systematic CT were performed after small lymph node EBUS-TBNA, we would see that minor pneumomediastinum, perivascular bleeding collections, or intramural hematomas near the great vessels would not be as rare as currently thought. In other words, this could occur more often and would most likely only be detected by CT. We agree with von Bartheld et al. [9] that the echobronchoscope may be useful to exclude complications when an intentional or accidental puncture of a mediastinal vessel occurs.

\section{Acknowledgement}

Partially supported by Research Intensification Activity from Fondo de Investigación Sanitaria (FIS). 


\section{References}

$D_{1}$ Groth SS, Andrade RS: Endobronchial and endoscopic ultrasound-guide fine-needle aspiration: a must for thoracic surgeons. Ann Thorac Surg 2010;89:S2079-S2083.

$\checkmark 2$ Falcone F, Fois F, Grosso D: Endobronchial ultrasound. Respiration 2003;70:179-194.

3 Varela-Lema L, Fernández-Villar A, RuanoRavina A: Effectiveness and safety of endobronchial ultrasound-transbronchial needle aspiration: a systematic review. Eur Respir J 2009;33:1156-1164.

4 Gross A, Diacon AH: Cardiac tamponade following transbronchial needle aspiration. Respiration 2011;82:56-59.
Huang CT, Chen CY, Ho CC, Yu CJ: A rare constellation of empiema lung abscess and mediastinal abscess as a complication of endobronchial ultrasound-guided transbronchial needle aspiration. Eur J Cardiothorac Surg 2011;40:264-265.

6 Parker KL, Bizekis CS, Zervos MD: Severe mediastinal infection with abscess formation after endobronchial ultrasound-guided transbronchial needle aspiration. Ann Thorac Surg 2010;89:1271-1272.

7 Liberman M, Duranceau A, Martin J, Thiffault V, Ferraro P: Major airway laceration secondary to endobronchial ultrasound transbronchial lymph node biopsy. J Bronchol Intervent Pulmonol 2010;17:264-265.
8 Vincent B, Huggins JT, Doelken P, Silvestri G: Successful real-time endobronchial ultrasound-guided transbronchial needle aspiration of hilar lung mass obtained by traversing the pulmonary artery. J Thorac Oncol 2006; 1:362-364.

$\checkmark 9$ von Bartheld MB, Rabe KF, Annema JT: Transaortic EUS-guided FNA in the diagnosis of lung tumors and lymph nodes. Gastrointest Endosc 2009;69:345-349. 